Document downloaded from:

http://hdl.handle.net/10251/50881

This paper must be cited as:

Torregrosa Jaime, B.; Corberán Salvador, JM.; Muller; Vasile, C.; Muller, C.; Risser, M.; Payá Herrero, J. (2014). Sizing of a reversible magnetic heat pump for the automotive industry. International Journal of Refrigeration. 37:156-164.

doi:10.1016/j.jirefrig.2013.06.018.

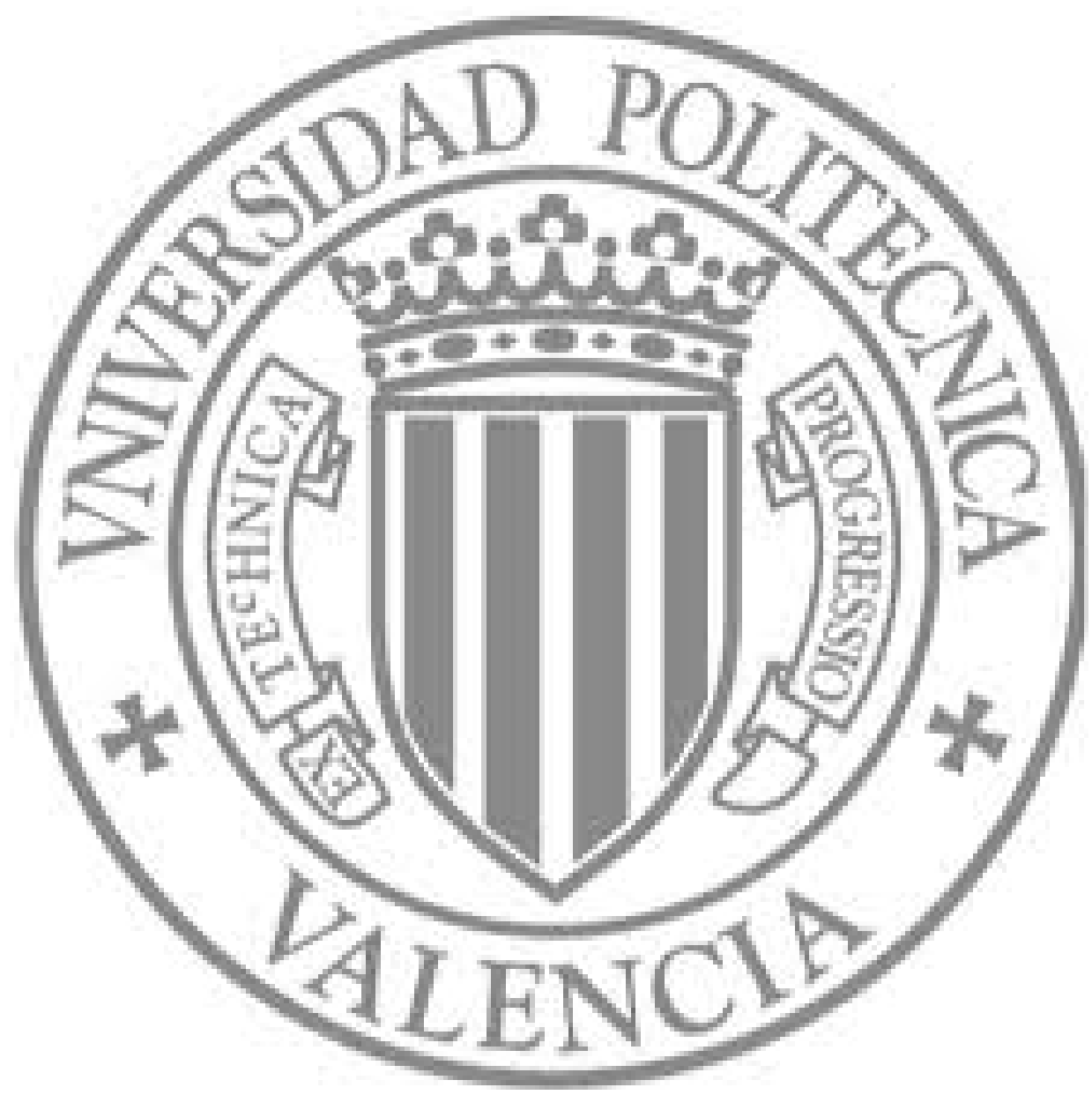

The final publication is available at

http://dx.doi.org/10.1016/j.jirefrig.2013.06.018

Copyright Elsevier 


\title{
Sizing of a reversible magnetic heat pump \\ for the automotive industry
}

\author{
B. Torregrosa-Jaimea , J.M. Corberána, C. Vasile ${ }^{b}$, \\ C. Muller ${ }^{c}$, M. Risser ${ }^{b}$, J. Payáa, ${ }^{*}$
}

a Instituto de Ingeniería Energética (IIE), Universitat Politècnica de València Camino de Vera s/n, Edificio 8E cubo F planta 5, 46022 Valencia, Spain

b LGeCo, National Institute of Applied Sciences (INSA), 24 Bd de la Victoire, 67084 Strasbourg Cedex, France

${ }^{c}$ Cooltech Applications, Impasse Antoine IMBS, 67810 Holtzheim, France

\section{Abstract}

This paper focuses on the design of an innovative air-conditioning system, namely a magnetocaloric air-conditioner for an electric minibus. An integrated design of the complete system is necessary, as the hot and cold side of the regenerator will work under dynamic conditions which depend on the instantaneous thermal load in the cabin.

In order to assist the design of the system, a dynamic model has been developed for the cabin, the hydraulic loops and heat exchangers, and the magnetocaloric unit. This paper presents (i) a description of the dynamic models, (ii) an analysis of the operating conditions of the magnetocaloric unit and (iii) a discussion on the design of the magnetocaloric air-conditioner. The results show that 
the electric minibus requests $1.60 \mathrm{~kW}$ of cooling power over a span of $37 \mathrm{~K}$ in cooling mode, and $3.39 \mathrm{~kW}$ of heating power over a span of $40 \mathrm{~K}$.

Keywords: Magnetic refrigerator, Heat pump, Automobile, Air conditioning, Modelling

${ }^{*}$ Corresponding author. Tel: +34 963879910; Fax: +34 963877272;

E-mail address: jorpaher@iie.upv.es (J. Payá)

\begin{tabular}{|c|c|c|c|}
\hline \multicolumn{4}{|c|}{ NOMENCLATURE } \\
\hline \multicolumn{2}{|c|}{ Acronyms } & \multicolumn{2}{|c|}{ Greek symbols } \\
\hline AMR & Active magnetic regenerator & $\Delta T_{\text {span }}$ & Temperature span (K) \\
\hline COP & Coefficient of performance (-) & $\varepsilon$ & Effectiveness (-) \\
\hline HTF & Heat transfer fluid & $\mu_{0}$ & Vacuum magnetic \\
\hline & & & permeability $\left(\mathrm{VsA}^{-1} \mathrm{~m}^{-1}\right)$ \\
\hline MCE & Magnetocaloric effect & & \\
\hline MCM & Magnetocaloric material & Subscripts & \\
\hline $\mathrm{MHP}$ & Magnetic heat pump & air & Air \\
\hline NTU & Number of heat transfer units (-) & amb & Ambient \\
\hline$A C$ & Air-conditioning & cold & Cold side \\
\hline & & driv & Driver \\
\hline Symbols & & $\mathrm{e}$ & External \\
\hline$A$ & Area $\left(m^{2}\right)$ & EL & External loop \\
\hline B & Induction $(T)$ & hot & Hot side \\
\hline$C_{p}$ & Specific heat $\left(\mathrm{J} \mathrm{kg}^{-1} \mathrm{~K}^{-1}\right)$ & $\mathrm{i}$ & Internal \\
\hline $\mathrm{H}$ & Magnetic field $\left(\mathrm{Am}^{-1}\right)$ & IL & Internal loop \\
\hline
\end{tabular}




\begin{tabular}{|llll|}
\hline h & Specific enthalpy $\left(\mathrm{J} \mathrm{kg}^{-1}\right)$ & in & Inlet \\
I & Solar irradiation $\left(\mathrm{W} \mathrm{m}^{-2}\right)$ & mag & Magnetic \\
$\dot{m}$ & Mass flow rate $\left(\mathrm{kg} \mathrm{s}^{-1}\right)$ & pass & Passengers \\
$\dot{Q}$ & Thermal power $(\mathrm{W})$ & s & Supply \\
$\mathrm{RH}$ & Relative humidity (\%) & sat & Saturated \\
$\mathrm{T}$ & Temperature $\left({ }^{\circ} \mathrm{C}\right)$ & visco & Viscosity \\
$\mathrm{U}$ & Global heat transfer coefficient & & \\
& $\left(\right.$ W $\left.\mathrm{m}^{-2} \mathrm{~K}^{-1}\right)$ & & \\
$\mathrm{u}$ & Heat transfer fluid speed $\left(\mathrm{m} \mathrm{s}^{-1}\right)$ & & \\
$\mathrm{v}$ & Vehicle speed $\left(\mathrm{m} \mathrm{s}^{-1}\right)$ & & \\
$\dot{W}$ & Power $(\mathrm{W})$ & \\
\hline
\end{tabular}

\section{INTRODUCTION}

During the last decade, many experimental prototypes of magnetic refrigerators at room temperature have been developed with different configurations and operating conditions. A review of these machines was presented by Yu et al. (2010) and more recent advances can be found in publications from Tura and Rowe (2011), Engelbrecht et al. (2012), Tušek et al. (2013) and Jacobs et al. (2012). Research is currently focusing on improving the performance of such devices in order to make them competitive with conventional vapour-compression chillers. Most of the reported magnetic refrigerators are laboratory prototypes which are under development to increase both the thermal power and temperature span in order to fullfill the 
requirements of industrial applications. Nevertheless, very few of these prototypes have been designed for a practical application (Jacobs et al., 2012).

An interesting market-application of this technology is the air-conditioning (AC) in automobiles. The AC presents the highest power consumption of the auxiliary components of a vehicle, especially in electric cars, in which there is practically no waste heat available. In fact, if a reversible vapour-compression heat pump is used, the autonomy of the vehicle can be shortened by $10 \%$ in winter and by $15 \%$ in summer with respect to a vehicle with no climate control, under typical weather conditions (Clodic et al., 2011). In theory, magnetic cooling and heating technology is more efficient (Yu et al., 2010), thus it can potentially reduce the energy consumption of the $A C$ and hereby increase the vehicle autonomy.

However, this application is very challenging given the actual state-of-the-art. Installed thermal power in vehicles for AC ranges from 3 to $5 \mathrm{~kW}$ of cooling power and from 5 to $10 \mathrm{~kW}$ of heating power. Typical working temperatures in summer can be $5{ }^{\circ} \mathrm{C}$ at the cold side and $50^{\circ} \mathrm{C}$ at the hot side. In winter they can drop down to -10 ${ }^{\circ} \mathrm{C}$ and $40 \stackrel{\circ}{\circ}$ respectively. Thus, a span of around $60 \mathrm{~K}$ must be covered. Other important requirements for this target application in the vehicle industry include safety, minimum weight and limited power consumption.

Up to date, no prototype has reached such performances. Moreover, only few authors deal with high-power devices. One notable exception is the work by Jacobs et al. (2012), who reported a layered regenerator which delivered $2049 \mathrm{~W}$ of cooling power at zero span and a peak performance of $1704 \mathrm{~W}$ over a span of $11.1 \mathrm{~K}$ with a COP of 2.24. Another example can be found in recent work from Engelbrecht et al. (2012), who built a prototype which worked with gadolinium and achieved $1010 \mathrm{~W}$ of cooling power at zero span and a maximum span of $25 \mathrm{~K}$ with no load. These recent 
results indicate that the performance is continuously improving. Current systems are still below the requirements for automotive applications but it is feasible to reach the required power on a mid-term, by means of an integrated design of the whole system.

The European project ICE aims to demonstrate the feasibility of magnetic heat pumps for the $A C$ of an electric minibus (ICE, 2013). This paper focuses on the first steps of the design of this innovative system which is mainly constrained by the volume, weight, the thermal demand and the working temperatures. The latter are key points in the design of a magnetic heat pump (MHP) or magnetic refrigerator (MR) (Tura and Rowe, 2011). Hence, it is essential to size the system properly and predict the effect of variable conditions on the external loops of the regenerator. This paper presents novel results in the overall approach which is followed, by means of a model of the complete vehicle as well as the AMR. Although this industrial application in the automotive industry requires a specific design, the same modelling approach can also be applied for the AC of spaces with other thermal loads.

\section{SYSTEM MODELLING}

An overall model of the magnetic $A C$ system of the electric minibus has been developed in order to assist its design and optimize its performance. This system contains two MHPs, one at the front of the vehicle (driver's region) and other at the rear (passengers' region). Given the state-of-the-art of magnetic refrigeration, and as discussed in the introduction, it seems more feasible to reach the required thermal powers for the vehicle if two MHPs are installed rather than only one. This approach 
also enables to reach different thermal comfort conditions, one in the driver region and one in the rear depending on the occupation of the vehicle.

The MHPs provide cooling or heating power to the minibus' cabin by means of the internal hydraulic loops, and reject or extract heat from the environment through the radiator of the vehicle. The system is electronically controlled to reach and maintain comfort conditions ( $\mathrm{T}$ and $\mathrm{RH}$ ) inside the cabin. A layout of the system with the main variables that determine its operation is shown in Fig. 1.

The overall model consists of physical-based dynamic models of every component, essentially a thermal model of the cabin, the MHPs and the thermal power distribution loops. These components are linked through the return and supply air and water flows, and interact under dynamic conditions imposed by the external environment and the control settings. In the next paragraphs, the models of each component are described.

\subsection{Cabin}

The cabin model (Torregrosa-Jaime, 2011; Torregrosa-Jaime et al., 2011) simulates the thermal behaviour of the minibus' cabin, which is the space that must be air-conditioned by the MHPs. The model is zero-dimensional, so it calculates the mean temperature and relative humidity inside the vehicle for both the driver and the passenger region. Taking into account that this model is coupled to others in order to simulate the whole system, this approach allows reaching a compromise between a low computation time and a reasonable accuracy (Gado, 2006).

One of the novelties of this cabin model is that it is divided into two thermal zones. Since there are two MHPs, one at the passenger's region and other at the 
driver's region, it is convenient to distinguish between the temperatures in each zone. Moreover, as the occupation of the vehicle is very variable, this helps to create different comfort control criteria depending on the occupation of the passenger region. For each thermal zone, the model considers the thermal loads due to occupation, ventilation, auxiliaries and solar irradiance. It also takes into account the air circulation between zones.

The model is based on differential equations which represent energy and mass balances (Torregrosa-Jaime, 2011). The heat transfer mechanisms are modelled using the conductance method including the thermal inertia of the masses (cabin air, car body and internal masses such as seats and curtains). This approach can also be applied for other AC applications provided that there is experimental data available because the models have to be fully validated first, given the simplifications which are necessary to develop a zero-dimensional model. In this case, the parameters were calculated given the dimensions and characteristics of the vehicle which is a DAILY ELECTRIC minibus from IVECO-ALTRA. The model has been recently validated with an experimental warm-up and cool-down test (Torregrosa-Jaime et al., 2013a).

\subsection{Hydraulic loops and heat exchangers}

The hydraulic loops and the heat exchangers ensure the heat transfer in each side of the heat pump, as shown in Fig. 2. The heat transfer fluid is a $50 \%$ ethyleneglycol solution. The external loop includes the radiator, which interacts with the external environment. The internal loop contains the air-coolers, which warm up or cool down the air inside the cabin of the vehicle depending on the season. 
The model of the loops determines the supply and return temperatures of the MHP by means of energy balances. The inertia and heat losses in the pipes have been considered. The thermal power of each heat exchanger is calculated by means of the effectiveness method (Eq. (1)), considering that air is the fluid with the minimum heat capacity. The enthalpy has been used instead of the temperature in order to take into account both latent and sensible changes in the air side.

$$
\dot{Q}=\dot{m}_{a i r} \cdot \varepsilon \cdot\left(h_{\text {air }, \text { in }}-h_{\text {sat,in }}\right)
$$

For designing purposes, a model of the heat exchanger based on the global heat transfer coefficient is more convenient than one based just on the effectiveness. In fact, using the first approach, the modelled heat exchanger can be scaled up and down and therefore its performance can be optimized. Considering the geometry of the heat exchangers in the minibus, which are mini-channel heat exchangers, the global heat transfer coefficient $(U)$ can be related to the effectiveness $(\varepsilon)$ with Eq. (2) (ESDU 86018, 1991), Eq. (3) and Eq. (4).

$$
\begin{gathered}
\varepsilon=1-\exp \left\{\frac{N T U^{0.22} \cdot\left[\exp \left(-C^{*} \cdot N T U^{0.78}\right)-1\right]}{C^{*}}\right\} \\
N T U=\frac{U A}{c_{p, a i r} \cdot \dot{m}_{\text {air }}} \\
C^{*}=\frac{C_{\min }}{C_{\max }}=\frac{c_{p, \text { air }} \cdot \dot{m}_{\text {air }}}{c_{p, w} \cdot \dot{m}_{w}}
\end{gathered}
$$


The effectiveness of a heat exchanger depends on its characteristics and on the temperatures and mass flow rates of the two fluids. Thus, experimental data is required to calculate the effectiveness. The experimental data was obtained from a series of tests on the radiator of the minibus (Torregrosa-Jaime et al., 2011). It should be noted that this modelling approach is applicable to any heat exchanger provided that the corresponding relation between $\varepsilon$ and NTU is given.

\subsection{Magnetic heat pump}

The principle of an Active Magnetic Regenerator (AMR) has been applied to design the MHP in order to reach the requirements in terms of thermal power and temperature span described in section 3. The AMR consists of a magnetocaloric material (MCM) matrix crossed by a coolant. Thus the reciprocating motion of the fluid in synchrony with the magnetic field change creates a thermal gradient in the matrix that can be used to exchange heat between the cold and hot ends. This thermal gradient allows reaching temperature spans which are several orders of magnitude higher than the adiabatic temperature change of the material (Barclay and Steyert, 1982).

A recent and extensive literature review on AMR numerical models is given by Nielsen et al. (2011). Some one-dimensional (1D) models allow obtaining a global and reliable overview of the system behaviour (Tagliafico et al., 2010; Vuarnoz and Kawanami, 2012). Engelbrecht and Bahl (2010) have also studied the effect of different magnetocaloric properties on the performance of a system by means of a 1D model. The numerical model used for the design of the present work has been recently described by Risser et al. $(2010,2013)$. Two key aspects in the design of an 
optimal AMR for this application are the frequency and the quantity of fluid passing through the AMR at each cycle.

The modelled AMR is based on magnetocaloric alloys whose Curie temperature is adapted for the requirements of this particular application. Indeed the Curie temperature of MCMs can be modified by tuning the proportion of additive elements in order to increase the efficiency and the temperature span of multi-material AMR (Richard et al., 2004). This approach can significantly improve the efficiency of the MHP compared to non-layered AMR (Egolf et al., 2012).

The selected materials for the simulations are in this study gadolinium-based alloys because they are the ones which have been characterized in more detail up to date, and they can be consequently simulated with more precision. The pure gadolinium magnetocaloric effect (MCE) is nevertheless sensibly weaker than magnetocaloric materials with a giant MCE such as for instance the MCM $\mathrm{Gd}_{5}\left(\mathrm{Si}_{2} \mathrm{Ge}_{2}\right)$ (Pecharsky and Gschneidner, 1997). Thus, the reachable performance is expected to increase within the next years with the recent advances on materials with a bigger MCE.

The MCM properties have been obtained as described in recent literature (Risser et al., 2012). The simulated MCMs are Gd-Tb alloys whose Curie temperature depends on the amount of $\mathrm{Tb}$ added to the $\mathrm{Gd}$.

The AMR matrix is composed of parallel plates as shown in the reference scheme of Fig. 3. The regenerator is composed of a stack of plates which contain the MCM and have a thickness of around 0.4-0.6 mm and they are placed parallel to the magnetic field. The plates have a typical spacing of $0.1-0.2 \mathrm{~mm}$ to ensure the circulation of the heat transfer fluid back and forth from the hot end to the cold end or vice versa. Such stacks can have a typical overall dimension of around $10 * 40 * 100$ 
$\mathrm{mm}$. The coolant is a mixture of water and glycol ( $30 \%$ volume). At each side of the AMR there is a heat exchanger which transfers heat to the hydraulic loops of the AC system.

The synchrony and the form of the applied varying magnetic field and of the fluid motion are selected to correspond with a rotating magnetocaloric system. This configuration is taken as reference for the future industrial MHP of Cooltech Applications (2013) because it seems to be the most efficient solution from both a technical and economical point of view (Kitanovski et al., 2012).

A magnetic field of $1.2 \mathrm{~T}$ is applied to AMR and is supposed to be uniform in space. This field value corresponds to reachable values with the currently available NeFeB permanent magnets used in industrial applications. The internal field $\left(\mathrm{H}_{\mathrm{i}}\right)$ and the induction (B) in the MCM caused by the applied field $\left(\mathrm{H}_{\mathrm{e}}\right)$ are calculated by means of a 3D model (Risser et al., 2013). Therefore, the demagnetizing field and the temperature distribution are taken into account when calculating the MCE. Fig. 4 shows some typical simulation results of the latter model. The results are completely dynamic and consequently, only the highest field values have been represented for the phase which includes heat transfer from the cold to the hot end of the regenerator. As may be inferred from Fig. 4, the internal field and the induction in the MCM are not uniform. Moreover, there are significant spatial differences along the regenerator and hereby the importance of working with a proper model which accounts for all of these aspects.

\section{RESULTS AND DISCUSSION}

\subsection{Sizing the MHP}


Once the main characteristics of the MHP have been outlined, the first step in the design process of a practical MHP is determining the composition of the AMR, in other words, the mass and Curie temperatures of the MCMs. To do so, the target cooling and heating powers as well as the working temperatures must be analysed.

Simulations of the cabin model were performed to calculate the thermal load inside the minibus in the ICE project design conditions for both summer and winter. Two working modes have been considered for each season. One is the steady-state with an average occupancy and the other is a pre-conditioning before start-up. The results are summarized in Table 1 . The design conditions set are not particularly severe, since the system is intended to be a demonstrator and the technology is still under development. This is the reason why the maximum thermal power requirements are relatively low.

As inferred from Table 1, the thermal load is unevenly distributed between the driver's and the passengers' regions. However, it is convenient to divide the total thermal power between the two MHPs. The simulations have shown that thermal comfort conditions can be achieved if half of the total load for a given working mode is overcome in each zone. In fact, inside the minibus there is not any physical barrier between the driver and passenger region, hereby the air can circulate freely. Since the most demanding working mode is the warm-up of the cabin during the preconditioning in winter, each MHP will be sized to overcome half of the total load (1520 W). The heat exchangers have been sized accordingly as well.

The temperatures which are reached in the receivers of the MHP are another key aspect to design the device, since they determine the selection of the MCMs. They are related to the supply and return temperatures to the MHP, which depend in 
turn on the design conditions and on the COP. The design process is iterative, since the COP of the MHP depends also on the working temperatures.

During the start-up of the system, the temperatures vary until the steady-state is reached. A preliminary simulation of the dynamics of the hydraulic system can help estimate the maximum temperature span. In a first iteration, since the COP of the MHP is unknown, the COP of a conventional heat pump has been taken as a reference. The goal of the project is to develop a system that can potentially replace a vapour-compression heat pump, so the MHP should reach at least a similar efficiency. Since the COP varies depending on the instantaneous return and supply temperatures, a conventional water-to-water heat pump model, adapted from recent work (Corberán et al., 2011) has been placed in the overall model in order to obtain the working temperatures.

The fluid mass flow rates in the hydraulic loops were calculated to achieve a typical maximum difference of $5 \mathrm{~K}$ between the return and the supply of the heat pump at steady-state conditions. Table 2 contains the corresponding values and a summary of the equilibrium temperatures.

The next figures show the dynamics of the AC system with the conventional heat pump. They are useful as a baseline to understand the dynamics of the system and design the MHP system.

Fig. 5 shows the equilibrium temperatures during the warm-up of the cabin in winter, as well as the total thermal power and the COP. Thermal comfort is reached after 45 minutes, as shown by the line that represents the temperature of the cabin in the driver zone. At this point, the conventional heat pump works with a COP of 2.2. 
In winter it is not necessary to dehumidify the air for comfort purposes. Nevertheless, the electric defogger on the windshield ensures that no fog appears on the inner part of the windshield.

Summer conditions are less demanding and hence the conventional heat pump presents a higher performance than the minimum requirements. In Fig. 6 it can be observed that the summer comfort goal with the conventional heat pump is reached in five minutes assuming a full occupation of the cabin (16 passengers plus the driver). Once the target temperature has been reached, the compressor cycles on and off in order to keep the temperature at the desired comfort level. At this point, the COP of the heat pump is 2.8. The temperatures in the hydraulic loops and the total thermal power and COP during the cool-down are also represented.

In summer, the relative humidity inside the cabin can be controlled by further cooling the air in order to dehumidify it, and then reheating up to the desired temperature. Thus, the MHP would be controlled to cool the air down until the requested humidity is reached, and then the air would be reheated using the waste heat from the system. Although $50 \% \mathrm{RH}$ was set as the comfort target in the ICE project (Table 1), acceptable summer conditions range from $25 \%$ to $65 \% \mathrm{RH}$ (ASHRAE, 2005). Since the RH is kept within this range in the simulations (Fig. 6), this issue has not been addressed in this preliminary sizing.

\subsection{Design of the MHP}

The design of the MHP as an iterative process enables the selection of the appropriate length of the AMR, the Curie temperatures positioning of the materials 
and the driving parameters. As explained in section 3, the maximum temperature span is obtained for the thermal gradient sized with the highest requirements for the device. The modelled MHP consists of a multi-material single stage system which leads to a possible drop in efficiency when the operating conditions are at a lower power level or at a lower temperature span than the maximum requirements. One possible option could even be to split the device into several independent stages to tune with a better ability the performance according to the operating conditions. Eight different MCMs with Curie temperatures have been selected following the method explained recently by the authors (Risser et al., 2013). This approach is based on rebuilding the magnetic, magnetocaloric and calorimetric data by means of an inverse approach in order to reach a good agreement with experimental data and at the same time ensure the conservation of the energy in the numerical model. The MCMs are distributed at regular intervals to cover the maximum span from about -6 ${ }^{\circ} \mathrm{C}$ to $46{ }^{\circ} \mathrm{C}$, as indicated in Table 2 . More specifically, the stacks are composed of Gd-Tb alloys with the following Curie temperatures: 2, 7, 12, 17, 22, 27, 32, and 37 ${ }^{\circ} \mathrm{C}$. The data are obtained on the basis of pure gadolinium data shifted in temperature and in amplitude through the process of reconstruction presented by Risser et al. (2012).

Fig. 7 represents the mean speed of the heat transfer fluid which flows along the channels of the regenerator. The evolution of the mean internal magnetic field is also shown $\left(\mu 0^{*} \mathrm{Hi}[\mathrm{T}]\right)$. The system consists of a rotary device with a fluid and magnetic synchronisation which has been optimised for this target application in the vehicle industry. Table 3 presents a summary of the parameters which have been selected in the simulations. The parameters refer basically to the geometry of the regenerator and to the operating frequency $(1.42 \mathrm{~Hz})$, which have been selected 
according to previous results described by Risser et al. (2013). In principle, the motion of the vehicle does not interfere the motion of the MHP, since in electric cars the AC system is powered by an independent electric motor, on the contrary to conventional thermal vehicles.

Fig. 8 shows the prediction of the cold thermal power pumped at the cold side and the hot thermal power provided by the MHP, both as a function of the output temperature span $\left(\Delta \mathrm{T}_{\text {span }}\right)$ centred on the comfort temperature of $20^{\circ} \mathrm{C}$. The exergetic consumption includes the magnetic work and the viscous dissipation. It must be noted that these results correspond to the use of gadolinium-based materials, since they are the ones whose characteristics have been measured precisely enough upto-date for modelling purposes. However, the power density obtained with this kind of alloys is lower than with other materials like the ones tested, for instance, in very recent prototypes (Jacobs et al., 2012). Therefore, there is a strong potential improvement which can be achieved by replacing $\mathrm{Gd}$ alloys with other magnetocaloric materials presenting better cooling capacities.

In Fig. 9 the COP and the ratio of COP on Carnot COP are represented, both as a function of the output temperature span ( $\left.\Delta \mathrm{T}_{\text {span }}\right)$ for the same conditions as Fig. 8. The optimal operating point for the efficiency does not correspond to the best power density operation point. Thus there is a trade-off depending on the available space in the vehicle.

Two important constraints in the automotive sector are the weight and cost of any new technologies. On the one hand, any additional weight increases the fuel consumption, and this is not negligible given the actual primary energy costs. The maximum overweight that could be accepted for this system compared with a conventional one would be of around $+20 \mathrm{~kg}$ (Di Sciullo, 2011; Torregrosa-Jaime et 
al., 2013b). Magnets represent a large part of the weight of a MHP, and thus its design must be carefully optimised for automotive applications. Nevertheless, when simulating the AMR in order to size the MHP, a valid approach is to consider the applied magnetic field to be uniform in space and to calculate the demagnetizing field within the MCM. The specific design of the magnet will be addressed in a further stage of the design.

On the other hand, costs are also a key issue for any industrial application. In the case of $A C$ systems for vehicles, the costs of standard heat pumps depend on their size and quality and range between 1.5 and $2.5 \mathrm{k} €$ for a production of 10000 pieces per year. Within the ICE project, the developed MHPs should be able to reach similar costs (Di Sciullo, 2011; Torregrosa-Jaime et al., 2013b).

\section{CONCLUSIONS}

This paper presents the first steps in the design of a reversible magnetic heat pump for the automotive industry. An overall model of a minibus has been developed, including a thermal model of the cabin, hydraulic loops, distribution system and the heat pump. The modelling approach can be applied to other AC systems provided that there is sufficient experimental data to build and validate the models. In the case of the minibus, the results show that a temperature span of $37 \mathrm{~K}$ and $40 \mathrm{~K}$ has to be overcome in summer and winter, with thermal powers of $1.60 \mathrm{~kW}$ and $3.39 \mathrm{~kW}$ respectively. The simulation results indicate that the required temperature span can be reached with Gd-Tb alloys with the Curie temperatures in the range from 2 to 37 
${ }^{\circ} \mathrm{C}$. Nevertheless, the performance is expected to be higher when using new magnetocaloric materials.

This overall approach has helped to pre-design the magnetic heat pump for an electric minibus. Currently, Cooltech Applications is assembling the first prototype for the vehicle based on the operating conditions defined in the present work. More experimental and modelling results are expected to be published soon.

\section{ACKNOWLEDGEMENTS}

This work has been supported by the European Commission under the $7^{\text {th }}$ European Community framework program as part of the ICE project "MagnetoCaloric Refrigeration for Efficient Electric Air-Conditioning", Grant Agreement no. 265434. B. Torregrosa-Jaime acknowledges the Spanish Science and Innovation Ministry (Ministerio de Ciencia e Innovación) for receiving the Research Fellowship FPU ref. AP2010-2160.

\section{REFERENCES}

ASHRAE, 2005. Thermal Comfort, in: ASHRAE (Ed.): 2005 ASHRAE Handbook Fundamentals SI Edition. ASHRAE, Atlanta, p. 18.12.

Barclay, J.A., Steyert, W.A., 1982. Active Magnetic Regenerator. U.S. Patent $4,332,135$.

Cooltech Applications, 2013. Available at <www.weenter.com>. 
Corberán, J.M., Finn, D.P., Montagud, C.M., Murphy, F.T., Edwards, K.C., 2011. A quasi-steady state mathematical model of an integrated ground source heat pump for building space control. Energy and Buildings 43, 82 - 92.

Clodic, D., Zgheib, E., Mortada, S., 2011. Impacts of heating and cooling on electrified vehicles, in: Proc. $4^{\text {th }}$ European Workshop MAC and Vehicle Thermal Systems 2011.

Di Sciullo, F., 2011. ICE Project: A magnetocaloric based mobile air-conditioning system, in: Proc. $4^{\text {th }}$ European Workshop MAC and Vehicle Thermal Systems 2011. Egolf, P.W., Vuarnoz, D., Gravier, L., Courret, G., 2012. The thermodynamics of devices with solid layered-bed magnetocaloric refrigerants. Int. J. Refrigeration 35, $1506-1517$.

Engelbrecht, K., Bahl, C.R.H., 2010. Evaluating the effect of magnetocaloric properties on magnetic refrigeration performance. J. of App. Physics 108, 123918.

Engelbrecht, K., Eriksen, D., Bahl, C.R.H., Bjork, R., Geyti, J., Lozano, J.A., Nielsen, K.K., Saxild, F., Smith, A., Pryds, N., 2012. Experimental results for a novel rotary active magnetic regenerator. Int. J. Refrigeration 35, 1498 - 1505.

ESDU 86018, 1991. Effectiveness - NTU Relationships for the Design and Performance Evaluation of Two-Stream Heat Exchangers, in: Engineering Science Data Unit 86018 with amendment A, ESDU International plc, London, pp. 92-107.

Gado, A., 2006. Development of a dynamic test facility for environmental control systems. PhD Thesis, University of Maryland, Maryland, USA, pp. $21-34$.

ICE, Magnetocaloric refrigeration for efficient electric air conditioning, 2013. < http://www.ice-project.webs.upv.es/>.

Jacobs, S., Auringer, J., Boeder, A., Chell, J., Komorowski, L., Leonard, J., Russek, S., Zimm, C., 2012. The performance of a large-scale rotary magnetic refrigerator. 
Proceedings of the $5^{\text {th }}$ International Conference on Magnetic Refrigeration at Room Temperature, Grenoble, France, pp. 421 - 428.

Kitanovski, A., Egolf, P.W., Poredos, A., 2012. Rotary magnetic chillers with permanent magnets. Int. J. Refrigeration 35, 1055-1066.

Nielsen, K.K., Tusek, J., Engelbrecht, K., Schopfer, S., Kitanovski, A., Bahl, C.R.H., Smith, A., Pryds, N., Poredos, A., 2011. Review on numerical modeling of active magnetic regenerators for room temperature applications. Int. J. Refrigeration 34, $603-616$.

Pecharsky, V.K., Gschneidner, K.A., 1997. Giant magnetocaloric effect in $\mathrm{Gd}_{5}\left(\mathrm{Si}_{2} \mathrm{Ge}_{2}\right)$. Physical Review Letters 78, $4494-4497$.

Richard, M.A., Rowe, A.M., Chahine, R., 2004. Magnetic refrigeration: Single and multimaterial active magnetic regenerator experiments. J. of App. Physics 95, 2146 2150.

Risser, M., Vasile, C., Engel, T., Keith, B., Muller, C., 2010. Numerical simulation of magnetocaloric system behaviour for an industrial application. Int. J. Refrigeration 33, $973-981$.

Risser, M., Vasile, C., Keith, B., Engel, T., Muller, C., 2012. Construction of consistent magnetocaloric materials data for modelling magnetic refrigerators. Int. J. Refrigeration 35, $459-467$.

Risser, M., Vasile, C., Muller, C., Noume, A., 2013. Improvement and application of a numerical model for optimizing the design of magnetic refrigerators. Int. J. Refrigeration 36, 950-957.

Tagliafico, G., Scarpa, F., Canepa, F., 2010. A dynamic 1-D model for a reciprocating active magnetic regenerator; influence of the main working parameters. Int. J. Refrigeration 33, $286-293$. 
Torregrosa-Jaime, B., Payá, J., Corberán, J.M., 2011. Modelling of mobile airconditioning systems for electric vehicles. Proceedings of the $4^{\text {th }}$ European Workshop MAC and Vehicle Thermal Systems 2011, Torino, Italy.

Torregrosa-Jaime, B., 2011. Modelling and analysis of an air-conditioning system for vehicles based on magnetic refrigeration. Master Thesis (in Spanish), Universitat Politècnica de València, Valencia, Spain.

Torregrosa-Jaime, B., Payá, J., Corberán, J.M., 2013a. Design of Efficient AirConditioning Systems for Electric Vehicles. SAE Int. J. Alt. Power 6(2), doi: 10.4271/2013-01-0864.

Torregrosa-Jaime, B., Payá, J., Corberán, J.M., Malvicino, C., Di Sciullo, F., 2013b. ICE Project: Mobile Air-Conditioning System Based on Magnetic Refrigeration. SAE Technical Paper 2013-01-0238, doi: 10.4271/2013-01-0238.

Tura, A., Rowe, A., 2011. Permanent magnet magnetic refrigerator design and experimental characterization. Int. J. Refrigeration 34, $628-639$.

Tušek, J., Kitanovski, A., Zupan, S., Prebil, A., Poredoš, A., 2013. A comprehensive experimental analysis of gadolinium active magnetic regenerators. Appl. Therm. Eng. $53,57-66$.

Vuarnoz, D., Kawanami, T., 2012. Numerical analysis of a reciprocating active magnetic regenerator made of gadolinium wires. Appl. Therm. Eng. 37, 388 - 395. Yu, B.F., Liu, M., Egolf, P.W., Kitanovski, A., 2010. A review of magnetic refrigerator and heat pump prototypes built before the year 2010. Int. J. Refrigeration 33, 1029 1060. 